\title{
Effects of diagnostic ultrasound on HSP70 expression in chorionic villi in rats during early pregnancy and the role of HSP70 in apoptosis in chorionic villi
}

\author{
HAITAO LIU $^{1 *}$, FENG HOU ${ }^{1}$, HAIYING LIANG ${ }^{1}$, GUOCHENG LIU ${ }^{1}$ and YUNFEI LI ${ }^{2 *}$ \\ ${ }^{1}$ Department of Obstetrics and Gynecology, Guangdong Provincial Women and Children's Hospital; \\ ${ }^{2}$ Department of Urology, The First Affiliated Hospital, Jinan University, Guangzhou, Guangdong 511400, P.R. China
}

Received June 14, 2013; Accepted September 6, 2013

DOI: 10.3892/ijmm.2013.1489

\begin{abstract}
The aim of this study was to investigate the effects of diagnostic ultrasound on chorionic villi during early pregnancy in rats through the examination of heat shock protein 70 (HSP70) expression and apoptosis in chorionic villi. Using TUNEL, RT-PCR, western blot analysis and immunohistochemistry, the extent of apoptosis and the gene/ protein expression of $\mathrm{HSP} 70, \mathrm{Bcl}-2$ and Bax was determined in the samples of the chorionic villus from the rats during early pregnancy. Compared with the unexposed group, there were clear signs that apoptosis had occurred in the villi exposed to ultrasound for $30 \mathrm{~min}$. The expression of HSP70 and Bax in the villi gradually increased with the extended duration of exposure to ultrasound, whereas the expression of Bcl-2 gradually decreased. Bcl-2 expression did not differ significantly between the group exposed for $10 \mathrm{~min}$ and the group exposed for $20 \mathrm{~min}$. Taken together, our data demonstrate that HSP70 may protect chorionic villi exposed to ultrasound by inhibiting apoptosis; however, its protective effects are limited over the extended duration of exposure to ultrasound. Thus, the prudent use of ultrasound during the early stages of pregnancy should be advocated.
\end{abstract}

\section{Introduction}

There has been controversy as to the safety of ultrasound for prenatal diagnostics (1). The potential biological effects of exposure to ultrasound on the embryo have gained increasing attention. Certain studies however, have stated that it may not damage the embryo, particularly during the first trimester of

Correspondence to: Dr Guocheng Liu, Guangdong Provincial Women and Children's Hospital, Xingnan Road 521-523, Panyu, Guangzhou, Guangdong 511400, P.R. China

E-mail: 1ht001018aaa@163.com

${ }^{*}$ Contributed equally

Key words: heat shock protein 70, ultrasound, chorionic villi, apoptosis, Bcl-2/Bax pregnancy and within diagnostic dose ranges (2). A previous study proved that diagnostic ultrasound can lead to apoptosis in chorionic villi during early pregnancy rats; however, due to the existence of anti-apoptotic factors, exposure to ultrasound at certain doses causes no real damage to chorionic villi (3). This suggests that exposure to diagnostic ultrasound deos not harm embryonic development. The potential damage of diagnostic ultrasound to the embryo still cannot be ignored in spite of human epidemiological studies not having found any detrimental effects on later fetal development (1).

In the process of embryonic development, a number of factors can protect the embryo from the adverse effects of the outside environment and ensure the normal development of the embryo. Heat shock protein 70 (HSP70) is closely associated with embryonic development and plays an important role in embryonic development (4). Previous studies have suggested that a number of mechanisms are involved in HSP70-mediated embryonic protection, including acting as a molecular chaperone involved in the folding of nascent and mis-folded proteins under non-stressful conditions, enhancing tolerance to heat stress, inhibiting apoptosis induced by variety of adverse factors, resisting damage from oxygen-free radicals and a role in immune protection (5).

To date, to our knowledge, there have been no studies on HSP70 expression in chorionic villi exposed to ultrasound. This study investigates the effects of exposure to diagnostic ultrasound on chorionic villi by examining HSP70 expression and apoptosis in chorionic villi during early pregnancy in rats exposed to ultrasound in order to provide an experimental basis for its safe application in clinical practice.

\section{Materials and methods}

Animal housing and specimen collection. A total of 70 adult female SPF Wistar rats (weight, 230-350 g; age, 12-16 weeks) were provided by the Animal Experiment Center of Southern Medical University, Guangzhou, China. The rats were then mated. They were fed a standard diet and maintained in a temperature- and light-controlled room $\left(21-27^{\circ} \mathrm{C}, 12 / 12 \mathrm{~h}\right.$ light/dark cycle, relative air humidity $40-70 \%$ and well ventilated with low noise) in accordance with the Animal Research Committee Guidelines of the Women and Children's Hospital 
of Guangdong Province. Pessaries were used to indicate the beginning of conception. The pregnant rats were exposed to diagnostic ultrasound approxiamtely 6-7 days after conception. The inspection procedure was as follows: the rats were fixed on the examination table. After local disinfection, the body surface corresponding to the uterus was exposed to an ultrasound instrument [Acuson 128XP/10: frequency, $2.5 \mathrm{MHz}$; spatial peak temporal average intensity (Ispta), $92 \mathrm{~mW} / \mathrm{cm}^{2}$; spatial peak, pulse average intensity (Isppa), $104 \mathrm{~mW} / \mathrm{cm}^{2}$; maximal intensity (Im), $\left.266 \mathrm{~mW} / \mathrm{cm}^{2}\right]$ and the existence of the embryo was confirmed. The rats were divided into four groups according to the duration of exposure: $0 \mathrm{~min}$ (group A), 10 min (group B), $20 \mathrm{~min}$ (group C) and $30 \mathrm{~min}$ (group D). The pregnant rats in each group were sacrificed $24 \mathrm{~h}$ after exposure and the chorionic villi were collected. Portions of the harvested tissues were flash frozen in liquid nitrogen for $30 \mathrm{~min}$ and cryopreserved, while the remaining tissues were fixed in $10 \%$ formaldehyde solution and paraffin embedded for future immunohistochemical staining and the detection of apoptosis. This study was approved by the Animal and Human Ethics Board of the Women and Children's Hospital of Guangdong Province.

\section{Methods}

Terminal deoxynucleotidyl transferase (TdT)-mediated dUDP nick-end labeling (TUNEL) assay. Apoptotic cells in the chorionic villi were detected using the In Situ Cell Apoptosis Detection kit (MK1024; Wuhan Boster Biological Engineering, Co., Ltd., Wuhan, China), with at least five replicates for each group. Reagents were prepared according to the manufacturer's instructions. The fixed chorionic villi were treated with $3 \% \mathrm{H}_{2} \mathrm{O}_{2}$ for $10 \mathrm{~min}$ at room temperature, washed three times and incubated in permeabilization buffer for $30 \mathrm{~min}$ at $37^{\circ} \mathrm{C}$. The chorionic villi were then washed three times in phosphate-buffered saline (PBS) and incubated with $50 \mu \mathrm{l}$ TUNEL reagents for $2 \mathrm{~h}$ at $37^{\circ} \mathrm{C}$ in the dark. The positive controls were incubated with $100 \mu 11000 \mathrm{U} / \mathrm{ml}$ DNase 1 (Sigma), which cleaves all DNA, for $20 \mathrm{~min}$ at $37^{\circ} \mathrm{C}$ and washed three times before TUNEL assay. The megative controls were incubated in PBS in the absence of TdT. The samples then were incubated in blocking reagent for $30 \mathrm{~min}$ at $37^{\circ} \mathrm{C}$ in the dark, followed by the addition of 1:100 anti-DIG-biotin and 1:100 streptavidin peroxidase for $30 \mathrm{~min}$ at $37^{\circ} \mathrm{C}$. After TUNEL assay, the chorionic villi were washed three times and counterstained with $50 \mu \mathrm{g} / \mathrm{ml}$ PI for $5 \mathrm{~min}$ at room temperature to label all nuclei. Finally, the chorionic villi were mounted on glass slides with 1,4-diazabicyclo[2.2.2]octane (DABCO) and examined under a light microscope. The positive reactants were noted as brown particles localized in the nuclei. Positive criteria included separated and disorganized cells, morphology of apoptotic nuclei (pyknotic nuclei, condensation and margination of nuclear chromatin or nuclear fragmentation) and no inflammatory responses around the cell. Those positive cells without the morphology of apoptotic nuclei were not judged to be apoptotic cells unless their staining intensity strongly contrasted with background and the cells were singly distributed. Positive cells were counted by a double-blinded method. Ten non-overlapping fields were randomly observed on each slide. The percentage of positive cells was defined as the apoptotic index (AI).
Immunohistochemistry. Protein expression was detected using the the SP immunohistochemistry kit (Zhongshan Golden Bridge Biotechnology Co., Ltd., Beijing, China). Briefly, paraffin-embedded samples were sectioned $(4 \mu \mathrm{m})$, patched and dewaxed. Sections placed in $0.01 \mathrm{M}$ citric acid (pH 6.0) buffer were heated for $5 \mathrm{~min}$ for antigen retrieval and subsequently blocked for 10 min with $\mathrm{H}_{2} \mathrm{O}_{2}$. They were then incubated overnight at $4^{\circ} \mathrm{C}$ with primary antibodies, goat polyclonal anti-HSP70 antibody (sc-1060) at 1:100, mouse monoclonal anti-Bcl-2 antibody (sc-7382) at 1:100 and mouse monoclonal anti-Bax antibody (sc-7480) at 1:100 (all from Santa Cruz Biotechnology, Inc., Santa Cruz, CA, USA). After rinsing with PBS, biotinylated goat anti-rabbit IgG secondary antibodies were applied for $1 \mathrm{~h}$, followed by streptavidin peroxidase complexes for $30 \mathrm{~min}$. Diaminobenzidine was used as the final chromogen, and the sections were counterstained with hematoxylin. The slices with positive expression were used as the positive control. The negative control was established with the primary antibody replaced by PBS. All methods were performed according to the manufacturer's instructions. Positive staining presented as faint yellow, brown orange, or dark brown staining in the cytoplasm and/or nucleus of the cells. The expression of HSP70, Bcl-2 and Bax was semi-quantitatively analyzed by integrated optical density (IOD) calculated using Image-Pro Plus 6.0 software (Media Cybernetics, Inc., Rockville, MD, USA).

Observation under an electron microscope (EM). For observation under an EM, the placental tissue samples fixed in $3.0 \%$ glutaraldehyde were washed in cold $0.1 \mathrm{M}$ phosphate buffer ( $\mathrm{pH} 7.4$ ) for $3 \mathrm{~h}$ at $4^{\circ} \mathrm{C}$ and post-fixed in $1 \%$ osmium tetroxide, buffered with $0.1 \mathrm{M}$ phosphate, dehydrated in graded alcohols. The tissue was embedded in epoxy resin. Sections (1- $\mu$ m-thick) were obtained using an ultramicrotome, stained with uranyl acetate and lead citrate, and examined undr an EM (Philips Tecnai; Philips, Eindhoven, The Netherlands).

RT-PCR analysis. Villous tissue was homogenized and total RNA was then extracted using TRIzol reagent (Life Technologies Corp., Carlsbad, CA, USA) according to the manufacturer's instructions. RNA quality was checked by running on RNase-free, $1 \%$ agarose gel electrophoresis, and a UV300 type ultraviolet spectrophotometer (Unicam) calculating the spectrophotometric absorbance parameters of A260/A280 nm, where the ratio was between 1.8 and 2.0. RT-PCR was employed to determine the expression levels of the HSP70, Bcl-2, Bax genes using RT-PCR reagent kit (Takara Bio, Inc., Shiga, Japan). cDNA was prepared from the total RNA $(2.0 \mu \mathrm{g})$ using oligo(dT) primers. The total reaction system is $29 \mu \mathrm{l}$ including $4 \mu \mathrm{l}$ reverse transcription product as the template. The primers were designed on the basis of the published sequence of HSP70, Bcl-2, Bax and $\beta$-actin. Cycling conditions were $95^{\circ} \mathrm{C}$ for $10 \mathrm{~min}$ followed by 40 cycles at $95^{\circ} \mathrm{C}$ for $15 \mathrm{sec}, 59^{\circ} \mathrm{C}$ for $10 \mathrm{sec}$ and $72^{\circ} \mathrm{C}$ for $40 \mathrm{sec}$. Finally, elongation was completed at $72^{\circ} \mathrm{C}$ for $60 \mathrm{sec}$. For each PCR product the melting curve was determined and the curve analysis for each primer set showed only one peak per product. To determine the relative quantification of gene expression for the target and reference ( $\beta$-actin) genes, the comparative threshold cycle number $\left(2^{-\Delta \Delta \mathrm{Ct}}\right)$ method was used after a validation experiment demonstrated that the efficiencies of the target and reference $(\beta$-actin) genes were approximately equal. $\mathrm{Ct}$ values 
Table I. Oligonucleotide primers used for RT-PCR in this study.

\begin{tabular}{llll}
\hline Gene & \multicolumn{1}{c}{ Forward primer sequence (5'-3') } & \multicolumn{1}{c}{ Reverse primer sequence (5'-3') } & Authors/(Refs.) \\
\hline HSP70 & TTGTCCATGTTAAGGTTTTGTGGTATA & GTTTTTTTCATTAGTTTGTAGTGATGCAA & Le Masson and Christians (6) \\
Bcl-2 & CCTGTGGATGACTGAGTACC & GAGACAGCCAGGAGAAATCA & Sharma et al (7) \\
Bax & GTTTCATCCAGGATCGAGCAG & CATCTTCTTCCAGATGGT & Sharma et al (7) \\
$\beta$-actin & ATGGAATCCTGTGGCATCCA & TCCACACAGAGTACTTGCGCTC & Sharma et al (7) \\
\hline
\end{tabular}

define the threshold cycle of PCR at which amplified products were detected. $\Delta \mathrm{Ct}$ is the difference in the threshold cycles for targets and $\beta$-actin (internal control) (8). Each reaction was performed in triplicate and included a non-template control. The nucleotide sequences of the primers used in our study are presented in Table I.

Western blot analysis. Tissue homogenates were centrifuged and proteins were extracted. The concentration of protein in each sample was determined using the Bradford formula. The samples were then mixed directly with gel running buffer (Life Technologies Corp.). Proteins were resolved by $10 \%$ SDS-polyacrylamide gel electrophoresis and transferred onto polyvinylidene fluoride (PVDF) membranes (Life Technologies Corp.). The membranes were blocked for $1 \mathrm{~h}$ at room temperature and then incubated with primary antibodies (at 1:1,000 for anti-HSP70, 1:200 for anti-Bcl-2 and 1:200 for anti-Bax, respectively) at $4^{\circ} \mathrm{C}$ overnight, and a goatanti human $\mathrm{IgG}$ peroxide-labeled antibody was used together with the ECL chemiluminescent system for detection using a gel imaging analysis system (Bio-Rad). $\beta$-actin (Santa Cruz Biotechnology, Inc.) was used as the loading control.

Statistical analysis. Data were analyzed using SPSS17.0 statistical software. Quantitative values are presented as the means \pm standard deviation (SD). Significant differences were assessed by a one-way ANOVA analysis. Comparisons between groups were carried out using the LSD method except that the Dunnett T3 method was used for data with unequal variances. A P-value refers to a comparison of a measured parameter in the experimental group with that of the appropriate control. $\mathrm{P}<0.05$ was considered to indicate a statistically significant difference.

\section{Results}

Cell apoptosis. TUNEL-positive cells were mainly observed in the syncytiotrophoblast in chorionic villi, while they were also found in interstitial cells and vascular endothelial cells in the villi stroma, with a few in the cytotrophoblast. Compared with the unexposed pregnant rats, there was no significant change in $\mathrm{AI}$ in the groups exposed for 10 and $20 \mathrm{~min}$ ( $\mathrm{P}>0.05)$; however, the number of apoptotic cells in the group exposed for $30 \mathrm{~min}$ significantly increased $(\mathrm{P}<0.05)$ (Fig. 1).

Expression of proteins in chorionic villi by immunohistochemistry. HSP70 protein was mainly expressed in the cytoplasm. There were small amounts of HSP70 observed in the unexposed group, and the expression intensity of HSP70 gradually increased with the prolonged exposure time $(\mathrm{P}<0.05)$. Bcl-2 protein was mainly expressed in the cytoplasm. There was some amount of Bcl-2 in the unexposed group, and the expression of Bcl-2 significantly decreased following exposure to ultrasound $(\mathrm{P}<0.05)$, although the levels did not differ between the groups exposed for 10 and $20 \mathrm{~min}$ ( $\mathrm{P}>0.05)$. By contrast, the expression of Bcl-2 was almost not observed in the group exposed for $30 \mathrm{~min}$. Likewise, Bax protein was also mainly expressed in the cytoplasm. There were small amounts of Bax in the unexposed group, and the expression intensity of Bax gradually increased with the prolonged exposure time $(\mathrm{P}<0.05)$ (Fig. 2).

Ultrastructural findings. In the unexposed group, both the syncytiotrophoblast and cytotrophoblast cells were characterized by round nuclei, nuclear membrane integrity and an obvious nucleolus, in which chromatin was uniformly distributed. Additionally, in the cytoplasm, there are abundant Golgi complexes, scattered glycogen particles, round or oval mitochondria and a rough endoplasmic reticulum with the ribosomes adhering on their surface. The intercellular junctions were tight (Fig. 3A).

In the group exposed for $10 \mathrm{~min}$, both the syncytiotrophoblast and cytotrophoblast cells were still characterized by nuclei with a regular shape, a mildly swollen nuclear membrane and a still obvious nucleolus, in which chromatin was still uniformly distributed and nuclear pyknosis was occasionally observed. Additionally, in the cytoplasm, there were slightly decreased Golgi complexes and glycogen particles, round or oval but mildly swollen mitochondria and a mildly expanded rough endoplasmic reticulum with the ribosomes on the surface falling off. There were also occasional lipid droplets and vacuoles. The intercellular junctions were still tight. Generally, compared with the unexposed group, there were no significant differences in the structure of chorionic villi in the group exposed for $10 \mathrm{~min}$ (Fig. 3B).

In the group exposed for $20 \mathrm{~min}$, both the syncytiotrophoblast and cytotrophoblast cells were still characterized by nuclei with a regular shape, a mildly swollen nuclear membrane and a still complete nucleolus, in which chromatin was not very uniformly distributed, including a part of lumplike euchromatin and margination of heterochromatin and nuclear pyknosis was occasionally observed. Additionally in the cytoplasm, there were slightly decreased Golgi complexes and glycogen particles, round or oval but mildly swollen mitochondria and a mildly expansive rough endoplasmic reticulum with the ribosomes on the surface falling off. There were also slightly increased lipid droplets and vacuoles. The 

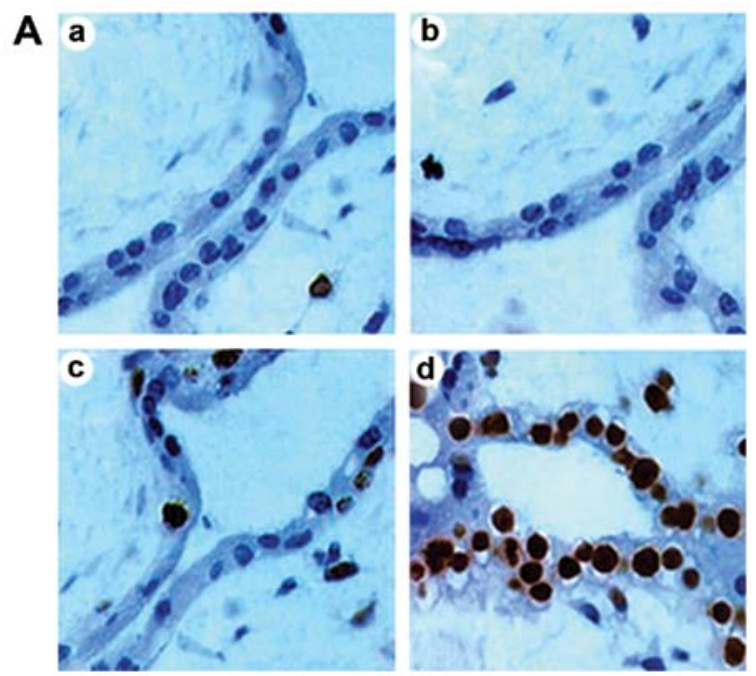

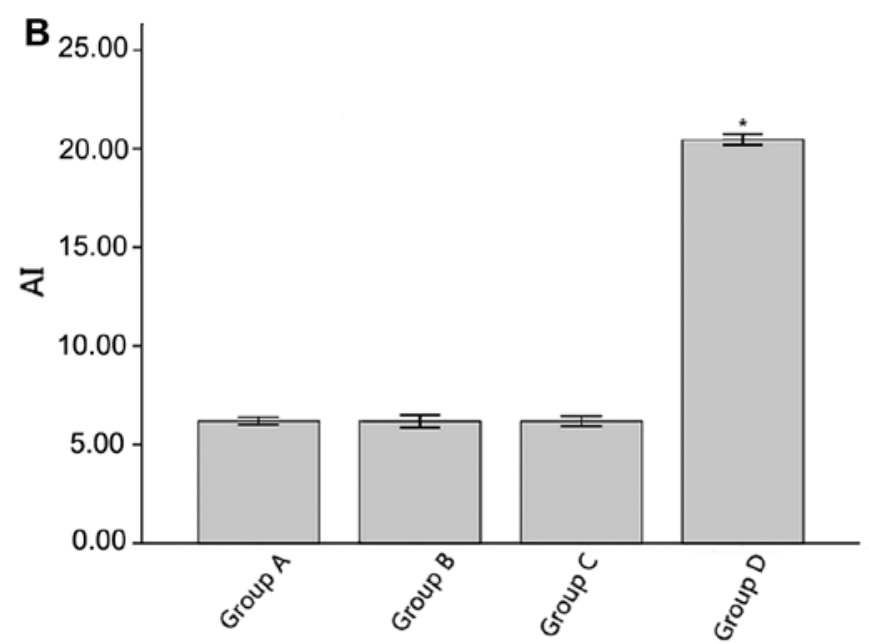

Figure 1. Apoptosis in chorionic villi following exposure to ultrasound. (A) Detection of apoptosis in chorionic villi in each group by TUNEL assay (magnification, x400). a, group A (unexposed group); b, group B (exposed for $10 \mathrm{~min}$ ); c, group C (exposed for $20 \mathrm{~min}$ ); d, group D (exposed for $30 \mathrm{~min})$. (B) Apoptotic index (AI) in chorionic villi in each group, determined by TUNEL assay (compared with other groups, ${ }^{\prime} \mathrm{P}<0.05$ ).
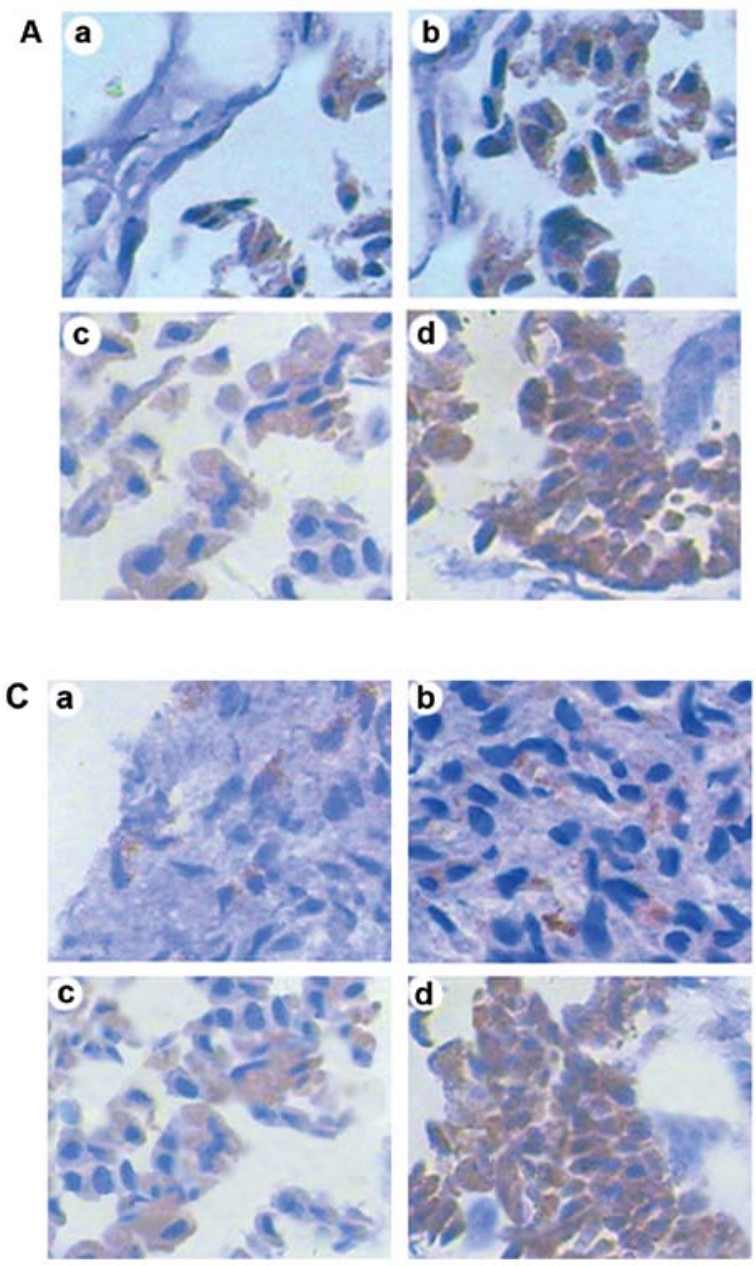
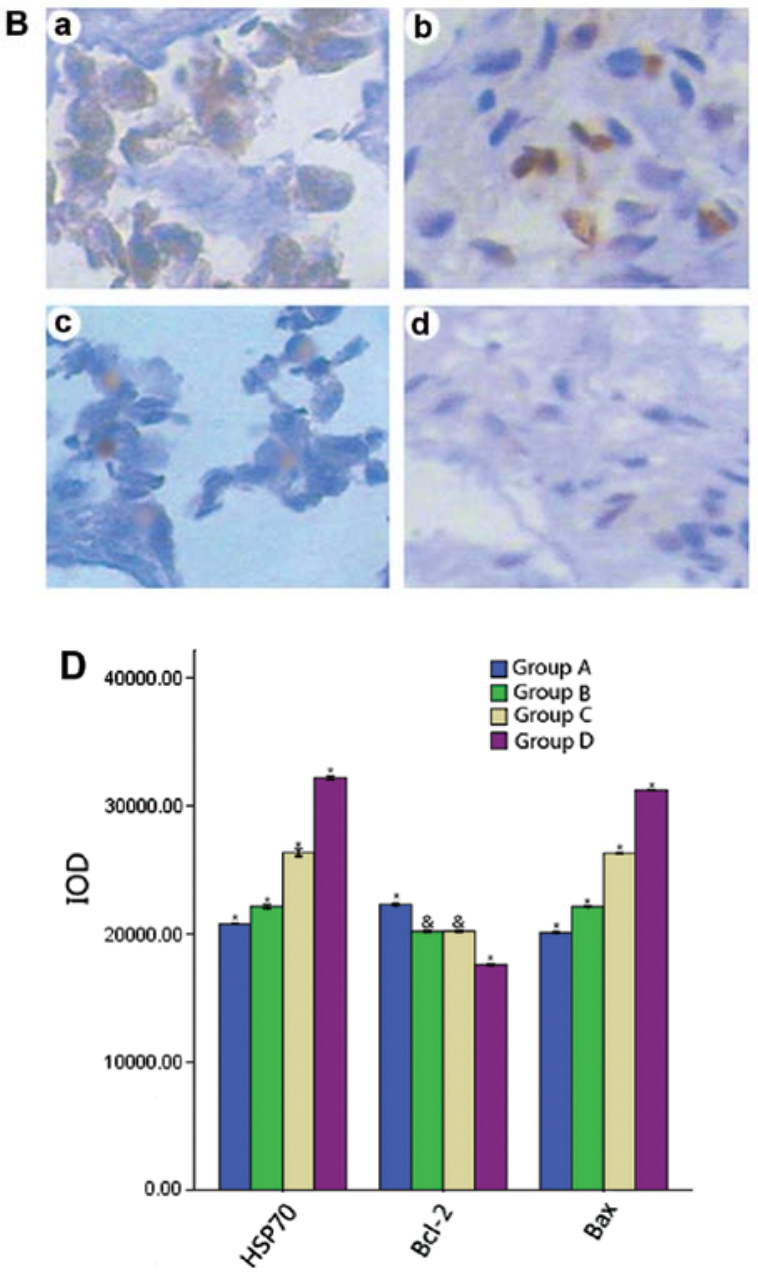

Figure 2. Protein expression analyzed by SP immunohistochemistry (magnification, $\mathrm{x} 400$ ). (A) Protein expression of HSP70 in chorionic villi in the different groups following exposure to ultrasound. There were small amounts of HSP70 in the unexposed group, and the expression intensity of HSP70 gradually increased with the prolonged exposure time. (B) Protein expression of Bcl-2 in the different groups Bcl-2 protein was mainly expressed in the cytoplasm. There was some amount of Bcl-2 in the unexposed group, and the expression of Bcl-2 significantly decreased following exposure to ultrasound, although the levels did not differ between the groups exposed for 10 and 20 min. The expression of Bcl-2 was almost not found in the group exposed for 30 min. (C) Protein expression of Bax in the different groups. Bax protein was also mainly expressed in the cytoplasm. There were small amounts of Bax in the unexposed group, and the expression intensity of Bax gradually increased with the prolonged exposure time. a, group A (unexposed group); b, group B (exposed for 10 min); c, group C (exposed for $20 \mathrm{~min}$ ); d, group D (exposed for $30 \mathrm{~min}$ ). (D) Integrated optical density (IOD) of each protein in the different groups (compared to other groups, "P $<0.05 ;$ * vs. \&, $\mathrm{P}<0.05 ; \&$ vs. \&, $\mathrm{P}>0.05$ ). 

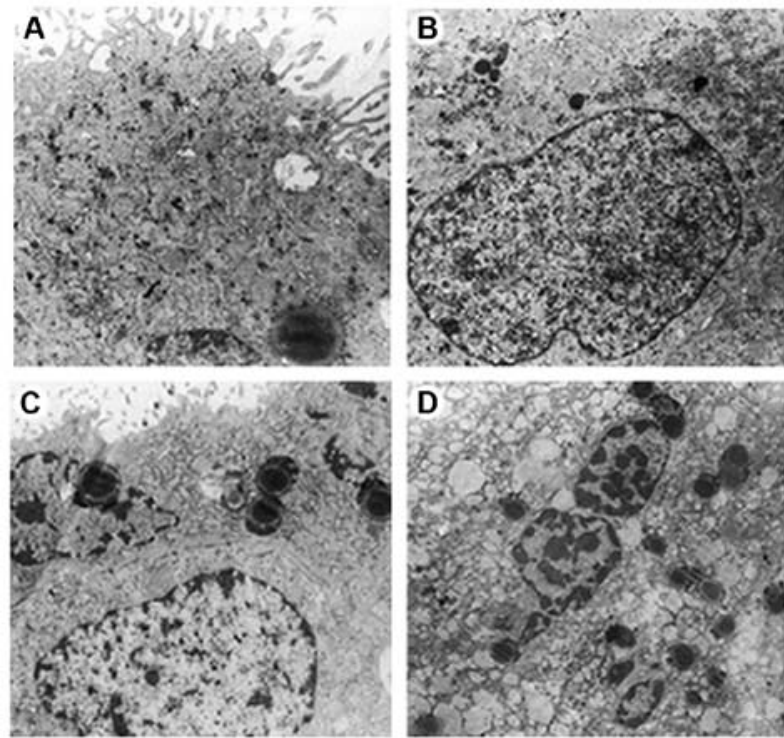

Figure 3. Ultrastructural observation of chorionic villi under an electron microscope following exposure to ultrasound. (A) group A (unexposed group); (B) group B (exposed for $10 \mathrm{~min}$ ); (C) group C (exposed for $20 \mathrm{~min}$ ); (D) group D (exposed for $30 \mathrm{~min})$.

gap between the intercellular junctions was slightly widened. Generally, compared with the unexposed group, there were no significant differences in the whole structure of chorionic villi in the group exposed for $20 \mathrm{~min}$ (Fig. 3C).

In the group exposed for $30 \mathrm{~min}$, both the syncytiotrophoblast and cytotrophoblast cells were characterized by nuclei with an irregular shape, an obviously swollen nuclear membrane and a nucleolus which had disappeared, in which chromatin was very unevenly distributed, including large amount of lump-like euchromatin and margination of heterochromatin and nuclear pyknosis was widely observed. Additionally in the cytoplasm, there were obviously decreased Golgi complexes and clustered glycogen particles, obviously swollen mitochondria with decreased and irregularly arranged cristae and a clearly expanded rough endoplasmic reticulum with the ribosomes on the surface falling off. There were also significantly increased lipid droplets and vacuoles. The gap between the intercellular junctions widened and looked blurry. Typical apoptotic bodies were observed, which contained some organelles, such as mitochondria. Generally, compared with the unexposed group, there were significant changes of in the structure of chorionic villi in the group exposed for $30 \mathrm{~min}$ that indicated damage (Fig. 3D).

Expression of HSP70, Bcl-2 and Bax $m R N A$. Compared with the unexposed group, the mRNA expression of HSP70 gradually increased with the prolonged exposure time $(\mathrm{P}<0.05)$. The mRNA expression of Bcl-2 significantly decreased following exposure to ultrasound $(\mathrm{P}<0.05)$, although there were no significant differences between the groups exposed for 10 and 20 min $(\mathrm{P}>0.05)$. The mRNA expression of $\mathrm{Bcl}-2$ was lower in the group exposed for $30 \mathrm{~min}$ than in the groups exposed for 10 and $20 \mathrm{~min}(\mathrm{P}<0.05)$. Likewise, the mRNA expression of Bax gradually increased with the prolonged exposure time $(\mathrm{P}<0.05)$ (Fig. 4).
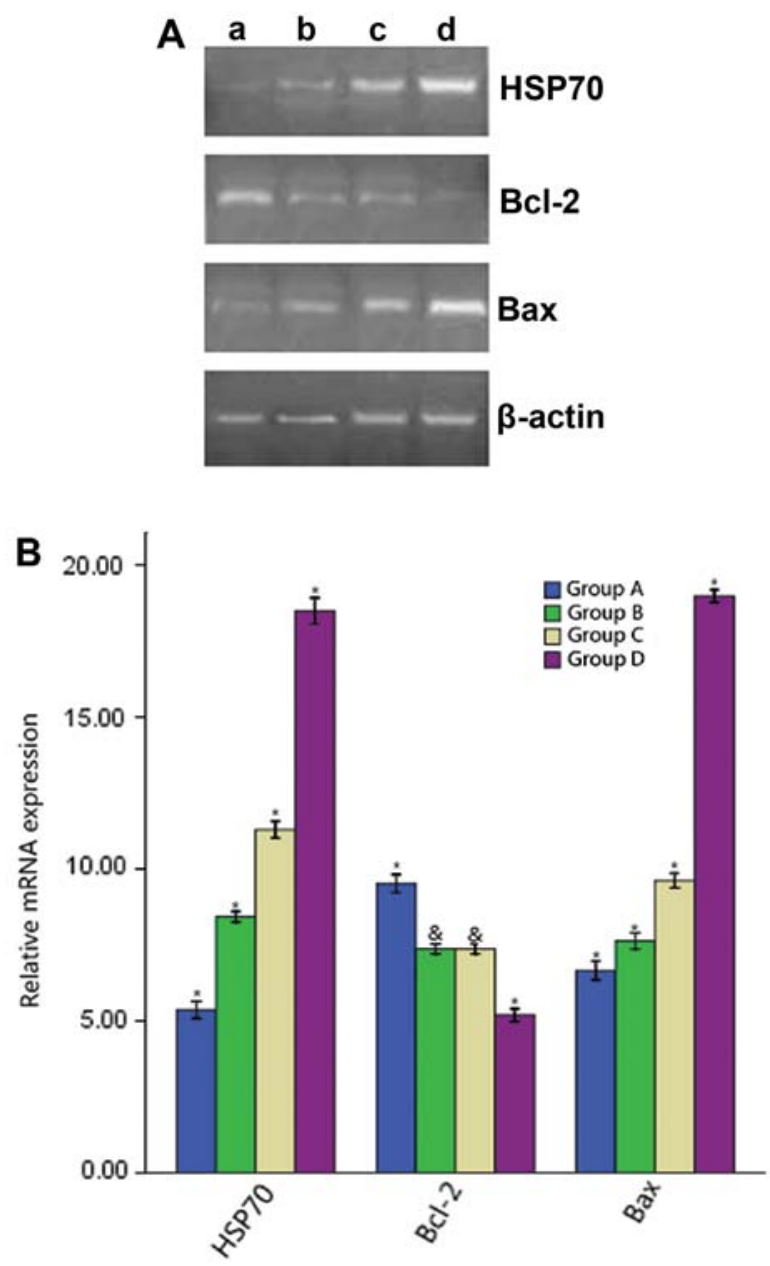

Figure 4. mRNA expression in chorionic villi in the different groups following exposure to ultrasound. (A) Results of RT-PCR. Lane a, group A (unexposed group); lane b, group B (exposed for $10 \mathrm{~min}$ ); lane c, group C (exposed for $20 \mathrm{~min}$ ); lane d, group D (exposed for $30 \mathrm{~min}$ ). (B) Relative mRNA expression of in the different groups (compared to other groups, ${ }^{*} \mathrm{P}<0.05$; * vs. $\&, \mathrm{P}<0.05$; $\&$ vs. \&, P>0.05).

Protein expression in chorionic villi as measured by western blot analysis. Compared with the unexposed group, the protein expression of HSP70 gradually increased with the prolonged exposure time $(\mathrm{P}<0.05)$. The protein expression of $\mathrm{Bcl}-2$ significantly decreased following exposure to ultrasound $(\mathrm{P}<0.05)$, although there were no significant differences between the groups exposed for 10 and $20 \mathrm{~min}$ ( $\mathrm{P}>0.05)$. The protein expression of Bcl-2 was lower in the group exposed for 30 min than in the groups exposed for 10 and $20 \mathrm{~min}(\mathrm{P}<0.05)$. Likewise, the protein expression of Bax gradually increased with the prolonged exposure time $(\mathrm{P}<0.05)$ (Fig. 5).

Ratio of Bcl-2/Bax in the different groups. With the prolonged exposure time, the ratio of $\mathrm{Bcl}-2 / \mathrm{Bax}$ in the different groups gradually decreased $(\mathrm{P}<0.05)$ (Fig. 6).

\section{Discussion}

The mechanisms of interaction of ultrasound with tissue that may lead to biological effects are often broadly divided into two categories, thermal and non-thermal (9). The absorp- 

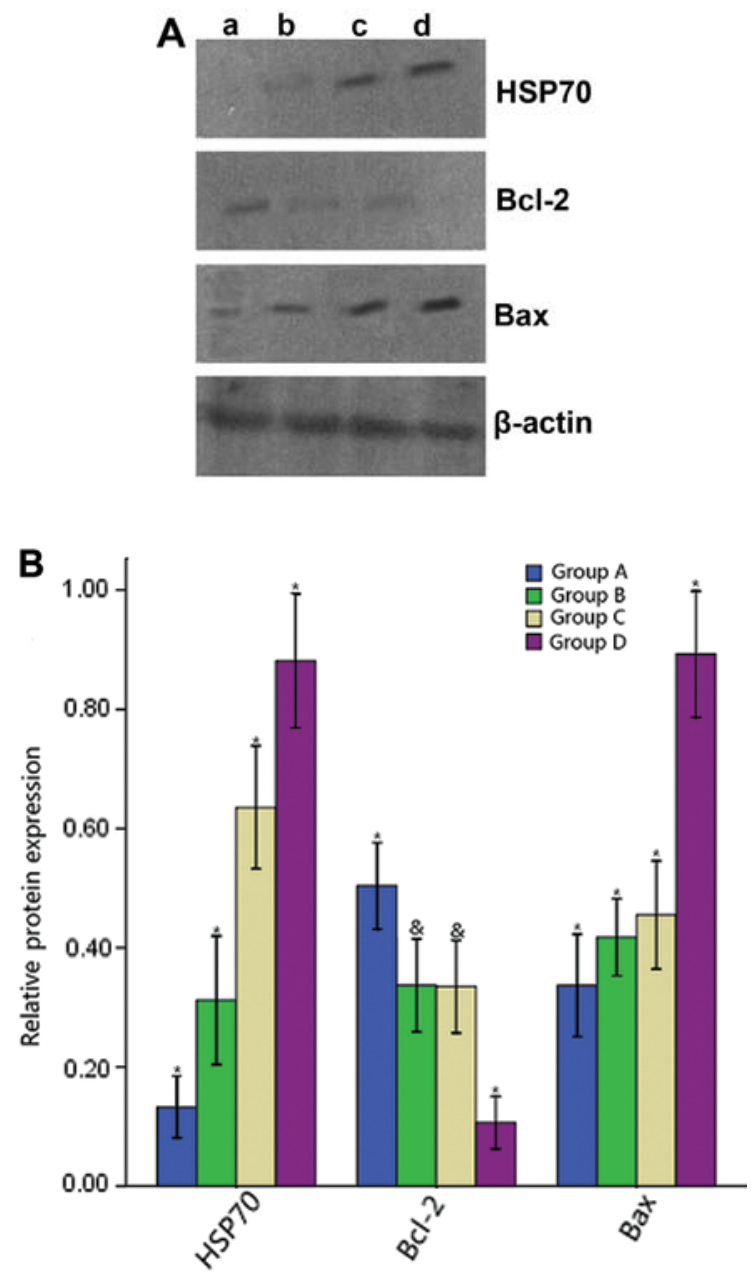

Figure 5. Protein expression of in chorionic villi in the different groups determined by western blot analysis following exposure to ultrasound Lane a, group A (unexposed group); lane b, group B (exposed for $10 \mathrm{~min}$ ); lane c, group C (exposed for $20 \mathrm{~min}$ ); lane d, group D (exposed for $30 \mathrm{~min}$ ). (A) Results of various proteins in each group. (B) Relative expression of various proteins in each group (compared to other groups, ${ }^{*} \mathrm{P}<0.05 ; *$ vs. \& $\mathrm{P}<0.05 ; \&$ vs. \&, $\mathrm{P}>0.05$.

tion of energy within tissue leads primarily to a rise in local temperature when a certain tissue is exposed to ultrasound. The magnitude of the thermal effect of ultrasound is related to organizational characteristics (including density, absorption coefficient, thermal conductivity and local blood perfusion), ultrasonic intensity and the time of ultrasonic exposure per unit volume, as well as ultrasonic pulse repetition frequency. For example, the thermal effects of ultrasound on bone are the most significant, and these effects are related to its dense structure and good conductivity, followed by the tendons and adipose tissue.

The non-thermal effects of ultrasound refer to the 'mechanical' effects of ultrasonic pressure waves through tissue, mainly the cavitation effect. The cavitation effect refers to various dynamic behaviors of gas bubbles in a certain sound field. The negative pressure in an ultrasonic pulse can draw gas out of a solution in tissue. These gas bubbles grow from existing nuclei in tissue. Once a bubble has been formed, it may expand and contract, in what are often termed breathing oscillations, in response to the pressure wave (non-inertial or stable cavita-

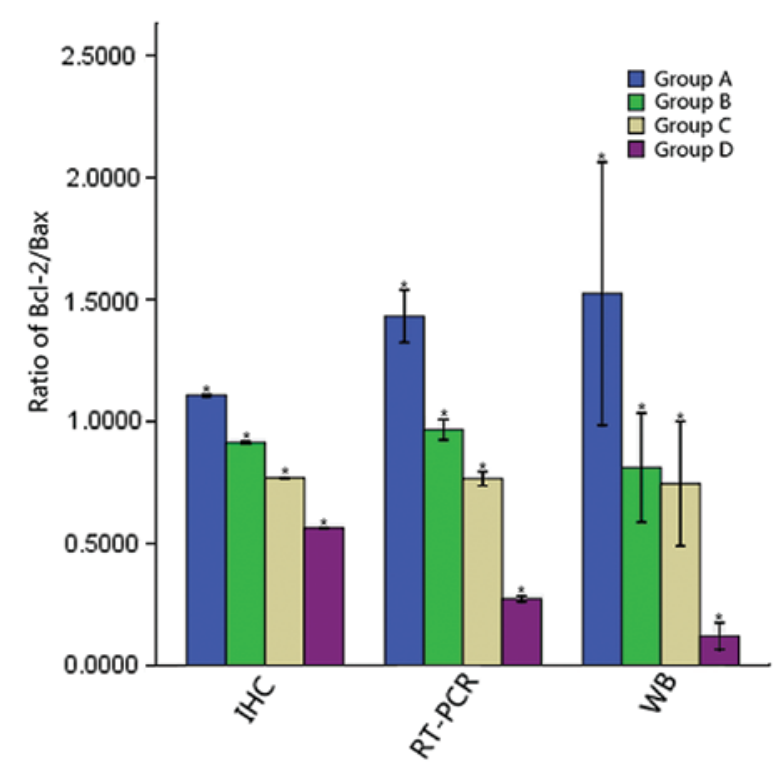

Figure 6. Ratio of Bcl-2/Bax in the different groups following exposure to ultrasound (compared to other groups, ${ }^{*} \mathrm{P}<0.05$; * vs. \&, $\mathrm{P}<0.05 ; \&$ vs. \&, $\mathrm{P}>0.05)$. IHC, immunohistochemistry; RT-PCR, reverse transcriptionpolymerase chain reaction; WB, western blot analysis. Group A (unexposed group); group B (exposed for $10 \mathrm{~min}$ ); group C (exposed for $20 \mathrm{~min}$ ); group D (exposed for $30 \mathrm{~min}$ ).

tion), or if at a size that is resonant for the drive frequency of the ultrasound beam, the bubble will greatly expand, followed by rapid contraction (inertial cavitation) and break up thereby damaging local tissue (1). To date, the cavitation effects of diagnostic ultrasound are generally thought not to have detrimental impacts on tissue while its damage to local tissue is mainly caused by the thermal effects $(2,10)$.

Previous studies on ultrasonic damage to the embryo have been frequently reported. An older study found that the body weight of newborns of cynomolgus macaques exposed to ultrasound with a $7.5 \mathrm{MHz}$ scanhead significantly decreased during the first three months after birth with short-term abnormal behavior (11). In the study by Ang et al (12), the authors found that the distribution of neurons of the cerebral neocortex in newborn mice differed significantly from control mice on the 10th day after birth when exposed to ultrasound wave for a total of $30 \mathrm{~min}$ or longer during the period of neuronal migration. Md Dom et al (13) suggested that parathyroid hormone (PTH) levels in rabbit fetal bodies exposed to ultrasound at different gestational stages were significantly decreased. A study on human chorionic villi indicated that the cleavage products of active caspase- 3 and cytochrome $c$ release were significantly increased in a time-dependent manner when exposed to transvaginal ultrasound in the first trimester of pregnancy (14). Moreover, the degree of damage diagnostic ultrasound has on the embryo differ at various developmental stages and tissues, in which embryo pre-implantation and the nervous system are the most sensitive stage and tissue, respectively $(2,15,16)$.

For the mammalian embryo to successfully complete development, it must not only incur proper timing of internal machinery, but also protect itself from potentially harmful external stimuli. To counterbalance these potential detriments, 
embryonic cells have some complex protective machinery (17). Early pregnancy is a key stage of embryonic development and the period during which the embryo is the most sensitive to risk factors. Thus, it is crucial to investigate the roles of this protective machinery during early pregnancy.

Heat shock proteins (HSPs) are highly conserved cellular stress proteins present in every organism from bacteria to man and are meant to protect cells from damage. HSPs can be broadly placed into five major families according to their molecular weight, amino acid sequence homologies and functions: HSP100 family, HSP90 family, HSP70 family, HSP60 family and the small HSP family (18). Previous studies have indicted that HSPs may play important protective roles in animal embryonic development. Zhang et al (19) found that more than ten HSP genes had differential expression between degenerate cattle embryos and blastocysts, whereas others were not expressed at all. This suggests the important roles of HSPs in fertilization and early development of the embryo. King et al (20) found that the small heat shock protein, p26, aids the development of encysted Artemia embryos, prevents spontaneous diapause termination and protects against stress. A study on embryonic stem cells (ESCs) suggested that HSP90 is essential for mouse ESC pluripotency by regulating multiple pluripotency factors, including Oct4, Nanog and signal transducer and activator of transcription 3 (STAT3) (21).

HSPs almost exist in all cells in an organism. The HSP70 family represents one of the most highly conserved classes of the HSPs, of which they are also the most abundant and appear the most when stress occurs (22). Under normal circumstances, there is a small amount of HSP70 expressed in the cytoplasm, whereas its synthesis rapidly increases with stress, the amount of which may account for $15 \%$ of total proteins in the stressed cells (23). The factors inducing HSP70 expression include changes in temperature, the presence of free oxygen radicals, viral and bacterial infections, heavy metals, ethanol and ischemia (24). HSP70 is crucial for the maintenance of cellular homeostasis during normal cell growth and for survival during and after various cellular stresses (25). In 1998, Neuer et al (26) indicated that mouse embryonic growth was markedly inhibited when monoclonal anti-HSP70 antibodies were supplemented to mouse embryos at the 2-cell stage, thus proving that HSP70 plays important roles in protecting normal embryonic development.

In the present study, we investigated the correlation between HSP70 and apoptosis in chorionic villi when the rat uterus was exposed to diagnostic ultrasound for different periods of time. The expression of HSP70 gradually increased with the prolonged exposure time. After $20 \mathrm{~min}$ of exposure, apoptosis and the ultrastructure of the exposed chorionic villi did not differ significantly from the unexposed pregnant rats, whereas the ultrastructure of the chorionic villi was remarkably damaged with obvious apoptosis following exposure to ultrasound for $30 \mathrm{~min}$.

Functionally, Bcl-2 and Bax are opposing genes, of which the former may maintain mitochondrial homeostasis, blocking the release of cytochrome $c$, inhibiting apoptosis, whereas the latter may increase mitochondrial permeability, enhance the release of cytochrome $c$ and promote apoptosis. The ratio of $\mathrm{Bcl}-2 / \mathrm{Bax}$ is usually used to judge the presence of apoptosis (27). Our results demonstrated that the expression of
Bcl-2 in chorionic villi significantly decreased when exposed to ultrasound, although the levels did not significantly differ between the groups exposed for 10 and $20 \mathrm{~min}$. By contrast, the expression of HSP70 gradually increased with the prolonged exposure time. Additionally, the ratio of Bcl-2/Bax gradually decreased with the prolonged exposure time, indicating a gradual increase in pro-apoptotic factors over time.

Based on the above results, it can by hypothesized that HSP70 may play important protective roles in rat chorionic villi exposed to ultrasound, protecting them from apoptosis. The thermal effects of ultrasound may increase the expression of HSP70, thereby protecting chorionic villi from apoptosis induced by heat stress. The regulation of Bcl-2 and Bax by HSP70 may be one of the important mechanisms by which HSP70 inhibits apoptosis. A previous study found that the gene transfer of HSP72 can protect the cornu ammonis 1 region of the hippocampus from global cerebral ischemia, partly by increasing Bcl-2 expression (28). Nagashima et al (29) suggested that HSP70 inhibits the activation of Bax through the suppression of the JNK/BIM pathway, thereby playing an anti-apoptotic role. The findings regarding the expression of Bcl-2 and Bax in the present study also indicate their regulation by HSP70. However, over a prolonged exposure to ultrasound, the pro-apoptotic factors become gradually predominant, which may prevail over the protective effects of HSP70. This results in the characteristic apoptotic and ultrastructural changes in the chorionic villi following exposure to ultrasound for $30 \mathrm{~min}$.

However, the overexpression of HSP70 also has detrimental effects on the embryo. Previous studies have suggested that HSP70 expression in the rat embryo in a stressed environment is frequently accompanied by the abundant co-expression of c-fos and the c-myc gene, which may interfere with the normal procedure of embryonic organogenesis. This may, however, also reflect damage, restoration and the regeneration of cells and tissues in the embryo $(30,31)$. In this study, as described above, the chorionic villi in the group exposed to ultrasound for $30 \mathrm{~min}$ presented apoptotic and ultrastructural changes characteristic of damage. Whether these changes were caused by the overexpression of HSP70 or other significantly increased pro-apoptotic factors need to be further investigated.

Additionally, apart from HSP70, a number of other factors can protect embryonic development. For example, the epidermal growth factor (EGF) plays an important role in protecting the cell or embryo from damage by promoting the synthesis of glutathione (GSH) (32). Stem cell factor (SCF) may improve the culture of mouse embryos exposed to unfavorable milieu by inhibiting apoptosis (33). Insulin-like growth factor 1 (IGF1) can protect the normal development of the pre-implantation bovine embryo by inhibiting apoptosis during heat stress (34). Thus, it may prove valuable to investigate the changes in these factors and their correlation with HSP70 in chorionic villi exposed to ultrasound.

Taken together, the data from this study suggest that HSP70 protects chorionic villi exposed to ultrasound by inhibiting apoptosisy. However, after prolonged exposure, apoptosis begins to occur as a result of an increase in pro-apoptotic factors or possibly HSP70-induced damage. Therefore, the prudent use of ultrasound during the early stages of pregnancy should be advocated. 


\section{Acknowledgements}

This study was supported by a grant from the Science and Technology Plan Program for Social Development of Guangdong Province (2011B061300093).

\section{References}

1. Gail ter Haar: Ultrasonic imaging: safety considerations. Interface Focus 1: 686-697, 2011.

2. Advisory Group on Non-ionising Radiation: Health effects of exposure to ultrasound and infrasound. Health Protection Agency, UK, 2010.

3. Li T, Shi HT, Yang WX and Xiong RP: Effect of diagnostic dose of color doppler ultrasound on apoptosis and Bcl-xl mRNA Caspase 3 mRNA express in rats embryos cells. Zhongguo Yi Xue Wu Li Xue Za Zhi 3: 1242-1245, 2009 (In Chinese).

4. Luft JC and Dix DJ: Hsp70 expression and function during embryogenesis. Cell Stress Chaperones 3: 162-170, 1999.

5. Hristova I: Role of heat shock proteins (Hsp) in human and mammalian fertilization and pregnancy. Part I. Akush Ginekol (Sofiia) 5: 45-49, 2012.

6. Le Masson F and Christians E: HSFs and regulation of Hsp70.1 (Hspalb) in oocytes and preimplantation embryos: new insights brought by transgenic and knockout mouse models. Cell Stress Chaperones 3: 275-285, 2011.

7. Sharma L, Kaur J and Shukla G: Role of oxidative stress and apoptosis in the placental pathology of Plasmodium berghei infected mice. PLoS One 3: e32694, 2012.

8. Livak KJ and Schmittgen TD: Analysis of relative gene expression data using real-time quantitative PCR and the 2(-Delta Delta C (T)) method. Methods 25: 402-408, 2001.

9. Fowlkes JB; Bioeffects Committee of the American Institute of Ultrasound in Medicine: American Institute of Ultrasound in Medicine consensus report on potential bioeffects of diagnostic ultrasound: executive summary. J Ultrasound Med 27: 503-515, 2008.

10. Sheiner E, Shoham-Vardi I, Hussey MJ, Pombar X, Strassner HT, Freeman $\mathbf{J}$ and Abramowicz JS: First-trimester sonography: is the fetus exposed to high levels of acoustic energy? J Clin Ultrasound 35: 245-249, 2007.

11. Tarantal AF and Hendrickx AG: Evaluation of the bioeffects of prenatal ultrasound exposure in the cynomolgus macaque (Macaca fascicularis). II. Growth and behavior during the first year. Teratology 39: 149-162, 1989.

12. Ang ES Jr, Gluncic V, Duque A, Schafer ME and Rakic P: Prenatal exposure to ultrasound waves impacts neuronal migration in mice. Proc Natl Acad Sci 103: 12903-12910, 2006.

13. Md Dom S, Abdul Razak HR, Ahmad Zaiki FW, Saat NH, Abd Manan K, Che Isa IN and Hashim UF: Ultrasound exposure during pregnancy affects rabbit foetal parathyroid hormone (PTH) level. Quant Imaging Med Surg 3: 49-53, 2013.

14. Zhang J, Zhou F, Song Y, Ying W and Zhang Y: Long dwell-time exposure of human chorionic villi to transvaginal ultrasound in the first trimester of pregnancy induces activation of caspase-3 and cytochrome $C$ release. Biol Reprod 67: 580-583, 2002.

15. Abramowicz JS, Barnett SB, Duck FA, Edmonds PD, Hynynen KH and Ziskin MC: Fetal thermal effects of diagnostic ultrasound. J Ultrasound Med 27: 541-563, 2008.
16. Ziskin MC and Barnett SB: Ultrasound and the developing nervous system. Ultrasound Med Biol 27: 875-876, 2001.

17. Driver AM and Khatib H: Physiology and Endocrinology Symposium: heat shock proteins: potentially powerful markers for preimplantation embryonic development and fertility in livestock species. J Anim Sci 91: 1154-1161, 2013.

18. Gupta SC, Sharma A, Mishra M, Mishra RK and Chowdhuri DK: Heat shock proteins in toxicology: how close and how far? Life Sci 86: 377-384, 2010.

19. Zhang B, Peñagaricano F, Driver A, Chen $H$ and Khatib H: Differential expression of heat shock protein genes and their splice variants in bovine preimplantation embryos. J Dairy Sci 94: 4174-4182, 2011.

20. King AM and MacRae TH: The small heat shock protein p26 aids development of encysting Artemia embryos, prevents spontaneous diapause termination and protects against stress. PLoS One 7: e43723, 2012.

21. Bradley E, Bieberich E, Mivechi NF, Tangpisuthipongsa D and Wang G: Regulation of embryonic stem cell pluripotency by heat shock protein 90. Stem Cells 8: 1624-1633, 2012.

22. Mayer MP and Bukau B: Hsp70 chaperones, cellular functions and molecular mechanism. Cell Mol Life Sci 62: 670-684, 2005.

23. Welch WJ: How cells respond to stress. Sci Am 268: 56-64, 1993.

24. Lindquist $S$ and Craig EA: The heat shock proteins. Annu Rev Genet 22: 631-677, 1988.

25. Rokutan K, Hirakawa T, Teshima S, et al: Implications of heat shock/stress proteins for medicine and disease. J Med Invest 44: 137-147, 1998.

26. Neuer A, Mele C, Liu HC, et al: Monoclonal antibodies to mammalian heat shock proteins impair mouse embryo development in vitro. Hum Reprod 4: 987-990, 1998.

27. Ola MS, Nawaz M and Ahsan H: Role of Bcl-2 family proteins and caspases in the regulation of apoptosis. Mol Cell Biochem 351: 41-58, 2011.

28. Kelly S, Zhang ZJ, Zhao H, Xu L, et al: Gene transfer of HSP72 protects cornu ammonis 1 region of the hippocampus neurons from global ischemia: influence of Bcl-2. Ann Neurol 52: 160-167, 2002.

29. Nagashima M, Fujikawa C, Mawatari K, Mori Y and Kato S: HSP70, the earliest-induced gene in the zebrafish retina during optic nerve regeneration: its role in cell survival. Neurochem Int 58: 888-895, 2011.

30. Sharp FR, Massa SM and Swanson RA: Heat-shock protein protection. Trends Neurosci 22: 97-99, 1999.

31. Tanaka K and Mizushima T: Protective role of HSF1 and HSP70 against gastrointestinal diseases. Int J Hyperthermia 25: 668-676, 2009.

32. Kim MK, Fibrianto YH, Oh HJ, et al: Effect of beta-mercaptoethanol or epidermal growth factor supplementation on in vitro maturation of canine oocytes collected from dogs with different stages of the estrus cycle. J Vet Sci 5: 253-258, 2004.

33. Glabowski W, Wiszniewska B and Kurzawa R: Protective potential of SCF for mice preimplantation embryos cultured in vitro in suboptimal conditions. J Assist Reprod Genet 25: 395-402, 2008.

34. Bonilla AQ, Oliveira LJ, Ozawa M, Newsom EM, Lucy MC and Hansen PJ: Developmental changes in thermoprotective actions of insulin-like growth factor-1 on the preimplantation bovine embryo. Mol Cell Endocrinol 332: 170-179, 2011. 